\title{
ÍNDICE DE SELEÇ̃̃O E ESTIMATIVA DE PARÂMETROS GENÉTICOS E FENOTÍPICOS PARA CARACTERÍSTICAS RELACIONADAS COM A PRODUÇÃO DE MILHO-VERDE
}

\author{
Index of selection and estimation of genetic and phenotypical parameters \\ for traits related with the production of vegetable corn
}

\author{
Fabricio Rodrigues ${ }^{1}$, Renzo Garcia Von Pinho ${ }^{2}$, \\ Carlos Juliano Brant Albuquerque ${ }^{3}$, Édila Vilela Resende Von Pinho ${ }^{2}$
}

\begin{abstract}
RESUMO
O Brasil é um dos maiores produtores de milho do mundo e possui grande potencial para a produção de milho verde. Além de agregar valor ao produto, a comercialização de milho verde cresceu muito nos últimos anos. Há escassez de informações sobre a avaliação e a obtenção de cultivares destinadas à produção de milho-verde e também sobre estudos genéticos das características envolvidas nessa produção. Objetivou-se, neste trabalho, estimar parâmetros genéticos e fenotípicos, a correlação genética, classificar e identificar híbridos de milho verde promissores ao mercado consumidor e avaliar a eficiência dos pesos econômicos atribuídos. Os resultados determinaram que a seleção terá sucesso, para as diferentes características de milho-verde, pois, a herdabilidade foi de alta magnitude para as características avaliadas. A avaliação da produtividade de espigas empalhadas (PEE) é suficiente para definir quais os híbridos apresentam o melhor desempenho para a produtividade de espigas comerciais (PEC), diâmetro (DIAM) e comprimento de espigas (COMP). Isso indica que as características PEC, DIAM e COMP podem ser descartadas da avaliação em programas de melhoramento de milho-verde. Os híbridos HS48, HS27, HS24, HS25, HS12 e HS45 acumularam um maior número de características desejadas em um único genótipo, com base no índice de seleção baseado na soma de postos, sendo os mais promissores para a produção de milho-verde. Os pesos econômicos utilizados foram capazes de distinguir e identificar os híbridos mais promissores para a produção de milho- verde e podem ser utilizados como critério de seleção em programas de melhoramento de milho-verde.
\end{abstract}

Termos para indexação: Zea mays L., programa de melhoramento, correlação genética, herdabilidade e consumo in natura.

\begin{abstract}
Brazil is one of the largest producers of corn of the world and it possesses great potential for vegetable corn production. Besides adding value to the product, the commercialization of vegetable corn increased in the last years. There is shortage of information about the evaluation and the acquisition of genotypes destined to the production of vegetable corn and also on genetic studies of the traits involved in this production. The objective of this work was to estimate the genetic and phenotypic parameters, and the genetic correlation, and to classify and identify hybrids of promising vegetable corn to the consumer market and to evaluate the efficiency of the attributed economical weights. The results determined that the selection will succeed, for the different traits of vegetable corn, because the heritability was of high magnitude to the appraised traits. Evaluation of husked ears yield (PEE) was enough to define which hybrid presents better commercial ear productivity, diameter and corn ear length. This indicates that those traits could be discarded in the selection process of genotypes of breeding programs for vegetable corn production. The hybrids HS48, HS27, HS24, HS25 and HS12 accumulated the largest number of desirable traits in a single genotype based on the sum of rank index. The used economical weights were efficient to distinguish and identify the most promising hybrid for vegetable corn production and they can be used as a selection criterion in programs of breeding of vegetable corn.
\end{abstract}

Index terms: Zea mays L., breeding programs, genetic correlation, heritability and consumption in natura.

(Recebido em 11 de maio de 2007 e aprovado em 14 de abril de 2010)

\section{INTRODUÇÃO}

O milho-verde é utilizado na alimentação sob diversas formas, podendo ser consumido in natura, cozido, assado ou processado. Alimento rico em carboidratos, sendo considerado como um alimento energético é também fonte de óleo e fibras e fornece pequenas quantidades de vitaminas E, B1, B2 e ácido pantotênico, além de alguns minerais, como o fósforo e o potássio (Matos et al., 2006).

A comercialização de milho-verde em Minas Gerais cresceu de, aproximadamente, 5 mil toneladas, em 1986, para mais de 20 mil toneladas, no ano de 2002. Apesar das oscilações nessa comercialização, é importante considerar que o aumento de $400 \%$ em 15 anos foi relevante, quando se compara com a evolução da produção e comercialização

1Universidade Estadual de Goiás/UEG - Ipameri, GO - fabriciorods@yahoo.com.br
'Universidade Federal de Lavras/UFLA - Departamento de Agricultura/DAG - Lavras, MG
${ }^{3}$ Empresa de Pesquisa Agropecuária de Minas Gerais/EPAMIG/CTNM - Nova Porterinha, MG

Ciênc. agrotec., Lavras, v. 35, n. 2, p. 278-286, mar./abr., 2011 
de outros produtos agrícolas no Estado (Pereira Filho et al., 2003).

Em programas de melhoramento, as estimativas de parâmetros genéticos e fenotípicos, são informações importantes e úteis para ajudar o melhorista nas tomadas de decisões. A herdabilidade permite antever a possibilidade de sucesso com a seleção, uma vez que ela reflete a proporção da variação fenotípica que pode ser herdada, ou seja, mede a confiabilidade do valor fenotípico como indicador do valor reprodutivo.

As correlações, também são importantes ao melhorista, pois, essas associações quantificam a possibilidade de ganhos indiretos por seleção em caracteres correlacionados. Além disso, caracteres de baixa herdabilidade têm a seleção mais eficiente, quando realizada sobre caracteres que lhe são correlacionados (Cruz \& Regazzi, 2001).

Especificamente com milho-verde há poucas informações disponíveis sobre parâmetros genéticos e fenotípicos e também sobre correlação entre características importantes para esse tipo de milho. A seleção com base em uma ou poucas características nem sempre é adequada para o melhorista. Dessa forma, a seleção simultânea de várias características oferece uma maior chance de sucesso com a seleção de genótipos promissores ao mercado. No caso do milho-verde, a rejeição pelo produto no mercado é favorecida quando as cultivares apresentam espigas com diâmetro, cor ou mesmo comprimento fora dos padrões desejados. A teoria do índice de seleção permite combinar as múltiplas informações contidas na unidade experimental e selecionar os genótipos superiores com base em um complexo de variáveis.

Objetivou-se, neste trabalho, estimar parâmetros genéticos e fenotípicos, a correlação genética, classificar híbridos de milho verde e identificar os mais promissores ao mercado consumidor, com base em várias características avaliadas.

\section{MATERIAL E MÉTODOS}

Foram utilizadas oito linhagens de milho, obtidas por meio de sucessivas autofecundações apresentando alto grau de homozigose e provenientes do programa de melhoramento da empresa Geneseeds Recursos Genéticos em Milho Ltda. As 8 linhagens, os 28 híbridos obtidos a partir do cruzamento entre essas linhagens, foram avaliados no ano agrícola de 2004/2005, em duas áreas experimentais, uma no município de Lavras e a outra no de Ijaci, ambos localizados na região Sul de Minas Gerais.
O delineamento experimental utilizado foi o de blocos casualizados com duas repetições, sendo avaliados 36 tratamentos (28 híbridos experimentais, 8 linhagens). As linhagens foram representadas pela letra $\mathrm{L}$ e os híbridos pelas letras HS. Para facilitar o estudo e a visualização, os híbridos foram representados de acordo com os pais que foram cruzados para formá-los, por exemplo, o híbrido HS27 é proveniente do cruzamento entre a linhagem L22 e L77.

As parcelas foram constituídas de quatro fileiras de $5 \mathrm{~m}$ com espaçamento de $0,8 \mathrm{~m}$ entre fileiras, sendo as duas fileiras centrais consideradas como úteis. As características avaliadas foram: produtividade de espigas empalhadas por hectare (PEE), produtividade de espigas comerciais por hectare (PEC), altura de planta (ALT), cor dos grãos (COR), porcentagem de massa (MASSA), diâmetro médio de espigas comerciais (DIAM) e comprimento de espigas comerciais (COMP). Todas essas avaliações foram realizadas de acordo com o método apresentado por Brant et al. (2008).

Todas as análises estatísticas e genéticas foram realizadas com o auxílio do programa computacional GENES. Os dados coletados dessas características foram submetidos à análise de variância individual e conjunta. Posteriormente, foram estimadas os parâmetros genéticos e fenotípicos, utilizando o procedimento apresentado por Vencovsky \& Barriga (1992), as correlações genéticas, utilizando o método apresentado por Cruz \& Regazzi (2001) e o índice de seleção baseado na soma de postos, utilizando o método de Mulamba \& Mock (1978).

Para a estimação do índice de seleção (I), os postos foram obtidos da seguinte maneira; todo genótipo recebeu um número de classificação para cada característica, adotando-se o critério de sempre utilizar o número 1 para a pior classificação da respectiva característica e, assim, sucessivamente até o número 28 para a melhor classificação. Desse modo, os indivíduos com classificação 28 foram aqueles com as menores médias para a característica ALT e as com maiores médias para as características PEE, PEC, MASSA, DIAM e COMP. Porém, para a característica COR, essa classificação foi feita de acordo com a nota de cor recebida na parcela (nota $1=$ classificação 7 , nota $1,5=$ classificação 6 e nota $2=$ classificação 5, e assim, sucessivamente, até a nota 4 ).

$$
I=\left(p_{1} \times r_{1}\right)+\left(p_{2} \times r_{2}\right)+\ldots+\left(p_{n} \times r_{n}\right)
$$

Em que:

I é o valor do índice para determinado híbrido; $r_{n}$ é a classificação do híbrido em relação ao n-ésima 
característica; $\mathrm{p}_{\mathrm{n}}$ é o peso econômico atribuído a n-ésima característica e n é o número da característica considerada no índice.

Os pesos econômicos foram atribuídos de acordo com o grau de importância de cada característica, onde PEE e PEC foram iguais a 1 , ALT foi igual a 0,2 , MASSA foi igual a 0,3, DIAM e COMP foram iguais a 0,5 e COR igual a 2. Posteriormente, os híbridos foram classificados com base nos índices obtidos.

\section{RESULTADOS E DISCUSSÃO}

Os resultados das análises de variância conjunta envolvendo os dois locais estão apresentados na Tabela 1. Para todas as características avaliadas, foram observadas diferenças significativas $(\mathrm{P} \leq 0,05)$, para a fonte de variação genótipos. Ficou constatada também, a diferença significativa entre os locais $(\mathrm{P} \leq 0,05)$ para a maioria das características avaliadas, com exceção da característica COR. Para a interação genótipos $\mathrm{x}$ locais não foram constatadas diferenças significativas, para a maioria das características, sendo que somente para a PEE e PEC ocorreu significância a $1 \%$ de probabilidade. Isso indica que a maioria dos genótipos apresentou comportamento semelhante nos dois locais para as características avaliadas.

Dentre os genótipos avaliados, somente os híbridos dos grupos "a" obtidos pelo teste de agrupamento de médias, em Lavras, apresentaram PEC acima de $12.000 \mathrm{~kg} \mathrm{ha}^{-1}$ (Tabela 2). Os híbridos HS24 e HS12 destacaram-se em Lavras, com produção de espigas comerciais superiores a esse valor. Resultados semelhantes foram obtidos por Pereira Filho et al. (2003) e Brant et al. (2008), utilizando os híbridos comerciais (AG 1051 e AG 4051) e híbridos simples experimentais. Vale ressaltar que as cultivares AG $1051 \mathrm{e}$ AG 4051 são as mais utilizadas para a produção de milhoverde no Brasil.

A característica ALT tem importância quando se considera a utilização da planta após a colheita, pois cultivares de menor porte são indicadas quando se deseja a incorporação dos restos culturais no solo, de forma que não prejudique o plantio posterior e que seja possível um maior número de colheitas por ano e por local (Bordallo et al., 2005). Os híbridos de maior altura, como, por exemplo, o HS28, HS13 e HS48, são indicados quando se pretende utilizar os restos culturais na alimentação de bovinos (Tabela 3).

Características, como COR, DIAM e COMP, merecem maior atenção do melhorista, pois a aparência das espigas destinadas à alimentação humana é de fundamental importância para a sua comercialização e exercem grande influência na aceitação do produto (Pereira Filho et al., 2003). Desse modo, as cultivares devem apresentar cor dos grãos entre as notas 1 (creme) e 2 (amarelo-claro), diâmetro de espiga igual ou superior a 3 $\mathrm{cm}$ e comprimento igual ou superior a $15 \mathrm{~cm}$.

Entre os genótipos avaliados, 18 para a característica cor e oito para o comprimento de espigas, não estavam dentro dos padrões exigidos pelo mercado de milho-verde. Entretanto, nenhum dos genótipos testados apresentou diâmetro inferior aos padrões exigidos para essa característica (Tabela 3).

As estimativas da variância fenotípica $\left(\sigma_{F}^{2}\right)$, variância ambiental $\left(\sigma_{E}^{2}\right)$ e variância genética $\left(\sigma_{G}^{2}\right)$ foram de maior magnitude em Lavras para as características ALT, PEE e COR (Tabela 4).

Tabela 1 - Resumo da análise de variância conjunta envolvendo os dois locais, para as características de altura de plantas (ALT), produtividade de espigas empalhadas (PEE), produtividade de espigas comerciais (PEC), porcentagem de massa (MASSA), cor dos grãos (COR), diâmetro (DIAM) e comprimento de espigas (COMP). UFLA, Lavras, MG, 2007.

\begin{tabular}{|c|c|c|c|c|c|c|c|c|}
\hline \multirow{2}{*}{ F.V } & \multirow{2}{*}{ GL } & \multicolumn{7}{|c|}{$\mathrm{QM}$} \\
\hline & & ALT & PEE & PEC & MASSA & COR & DIAM & COMP \\
\hline Genótipos & 35 & $0,313 * *$ & $4445731,36 * *$ & $23888211,26^{*}$ & $484,97 * *$ & $2,49 * *$ & $0,52 * *$ & $20,42 * *$ \\
\hline Locais & 1 & $15,16^{* *}$ & $948316286,11 * *$ & $376837633,94 * *$ & $929,41 * *$ & $1,00^{\mathrm{n} . \mathrm{s}}$ & $4,47 * *$ & $131,71 * *$ \\
\hline $\begin{array}{c}\text { Genótipos x } \\
\text { Locais }\end{array}$ & 35 & $0,5^{\mathrm{n} \cdot \mathrm{s}}$ & $44927225,10 * *$ & $8289692,69 * *$ & $92,98^{\mathrm{n} . \mathrm{s}}$ & $0,41^{\mathrm{n} . \mathrm{s}}$ & $0,12^{\mathrm{n} . \mathrm{s}}$ & $2,17^{\mathrm{n} . \mathrm{s}}$ \\
\hline Erro & 70 & 0,02 & 945330,50 & 1169200,50 & 40,87 & 0,38 & 0,07 & 1,19 \\
\hline Média & & 1,85 & 13481,29 & 5493,01 & 39,78 & 2,29 & 4,16 & 15,74 \\
\hline $\mathrm{CV} \%$ & & 8,43 & 11,77 & 20,67 & 16,06 & 27,02 & 6,81 & 7,47 \\
\hline
\end{tabular}

* e **- significativo, a $1 \%$ e $5 \%$ de probabilidade, pelo teste $\mathrm{F}$; n,s- não significativo. 
Tabela 2 - Produtividade de espigas empalhadas (PEE) e produtividade de espigas comerciais (PEC) de genótipos de milho avaliados em Lavras e Ijaci. UFLA, Lavras, MG, 2007.

\begin{tabular}{|c|c|c|c|c|c|c|c|c|}
\hline \multirow{3}{*}{$\begin{array}{c}\text { GENÓTIPOS } \\
\text { L11 }\end{array}$} & \multicolumn{4}{|c|}{ LAVRAS } & \multicolumn{4}{|c|}{ IJACI } \\
\hline & \multicolumn{2}{|c|}{$\operatorname{PEE}\left(\mathrm{Kg} \mathrm{ha}^{-1}\right)$} & \multicolumn{2}{|c|}{$\operatorname{PEC}\left(\mathrm{Kg} \mathrm{ha}^{-1}\right)$} & \multicolumn{2}{|c|}{$\operatorname{PEE}\left(\mathrm{Kg} \mathrm{ha}^{-1}\right)$} & \multicolumn{2}{|c|}{$\operatorname{PEC}\left(\mathrm{Kg} \mathrm{ha}^{-1}\right)$} \\
\hline & 9002,61 & $\mathrm{c}$ & 1700,92 & $\mathrm{e}$ & 7920,00 & $\mathrm{~b}$ & 1625,01 & $\mathrm{~b}$ \\
\hline HS12 & 20869,36 & $\mathrm{a}$ & 12231,28 & $\mathrm{a}$ & 12437,50 & $\mathrm{a}$ & 3828,13 & $\mathrm{~b}$ \\
\hline HS13 & 15513,89 & $\mathrm{~b}$ & 7471,24 & $\mathrm{~d}$ & 11444,12 & $\mathrm{a}$ & 5734,38 & $\mathrm{a}$ \\
\hline HS14 & 19631,46 & $\mathrm{a}$ & 9357,58 & $\mathrm{c}$ & 12448,70 & $\mathrm{a}$ & 6203,13 & $\mathrm{a}$ \\
\hline HS15 & 18540,88 & $\mathrm{a}$ & 9139,48 & $\mathrm{c}$ & 13556,06 & $\mathrm{a}$ & 6718,75 & $\mathrm{a}$ \\
\hline HS16 & 15885,15 & $\mathrm{~b}$ & 7705,93 & $\mathrm{c}$ & 11925,81 & $\mathrm{a}$ & 3617,19 & $\mathrm{~b}$ \\
\hline HS17 & 15708,33 & $\mathrm{~b}$ & 8242,91 & $\mathrm{c}$ & 12387,52 & $\mathrm{a}$ & 5875,00 & $\mathrm{a}$ \\
\hline HS18 & 19211,31 & $\mathrm{a}$ & 8557,54 & $\mathrm{c}$ & 11881,31 & $\mathrm{a}$ & 2695,32 & $\mathrm{~b}$ \\
\hline L22 & 9606,25 & $\mathrm{c}$ & 0,00 & $\mathrm{f}$ & 4232,07 & $\mathrm{c}$ & 869,53 & $\mathrm{~b}$ \\
\hline HS23 & 19232,19 & $\mathrm{a}$ & 10902,63 & $\mathrm{~b}$ & 11869,69 & $\mathrm{a}$ & 2570,32 & $\mathrm{~b}$ \\
\hline HS24 & 21168,75 & $\mathrm{a}$ & 13881,25 & $\mathrm{a}$ & 12491,90 & $\mathrm{a}$ & 2601,57 & $\mathrm{~b}$ \\
\hline HS25 & 20008,78 & $\mathrm{a}$ & 9864,87 & $\mathrm{~b}$ & 12904,81 & $\mathrm{a}$ & 5757,82 & $\mathrm{a}$ \\
\hline HS26 & 17979,99 & $\mathrm{a}$ & 9010,46 & $\mathrm{c}$ & 12361,91 & $\mathrm{a}$ & 3531,26 & $\mathrm{~b}$ \\
\hline HS27 & 21302,22 & $\mathrm{a}$ & 10939,91 & $\mathrm{~b}$ & 13706,90 & $\mathrm{a}$ & 5687,50 & $\mathrm{a}$ \\
\hline HS28 & 17915,62 & $\mathrm{a}$ & 8531,69 & $\mathrm{c}$ & 13828,41 & $\mathrm{a}$ & 3156,25 & $\mathrm{~b}$ \\
\hline L33 & 13855,03 & $\mathrm{~b}$ & 5607,68 & d & 9949,50 & $\mathrm{a}$ & 2923,00 & $\mathrm{~b}$ \\
\hline HS34 & 18076,35 & $\mathrm{a}$ & 6647,48 & d & 11625,54 & $\mathrm{a}$ & 6140,63 & $\mathrm{a}$ \\
\hline HS35 & 16617,54 & $\mathrm{~b}$ & 6820,86 & d & 9846,43 & $\mathrm{a}$ & 3375,01 & $\mathrm{~b}$ \\
\hline HS36 & 14163,50 & $\mathrm{~b}$ & 6904,41 & d & 10779,17 & $\mathrm{a}$ & 1851,57 & $\mathrm{~b}$ \\
\hline HS37 & 16695,79 & $\mathrm{~b}$ & 9278,04 & $\mathrm{c}$ & 9753,88 & $\mathrm{a}$ & 4000,01 & $\mathrm{~b}$ \\
\hline HS38 & 8799,13 & $\mathrm{c}$ & 5155,57 & $\mathrm{~d}$ & 7310,40 & $\mathrm{~b}$ & 2713,87 & $\mathrm{~b}$ \\
\hline L44 & 9158,66 & $\mathrm{c}$ & 275,00 & $\mathrm{f}$ & 5084,47 & $\mathrm{c}$ & 1152,35 & $\mathrm{~b}$ \\
\hline HS45 & 20139,43 & $\mathrm{a}$ & 9645,43 & $\mathrm{~b}$ & 14625,29 & $\mathrm{a}$ & 6609,38 & $\mathrm{a}$ \\
\hline HS46 & 16780,95 & $\mathrm{~b}$ & 8539,07 & $\mathrm{c}$ & 11838,71 & $\mathrm{a}$ & 4750,00 & $\mathrm{a}$ \\
\hline HS47 & 14713,82 & $\mathrm{~b}$ & 6854,56 & d & 12261,22 & $\mathrm{a}$ & 6539,07 & $\mathrm{a}$ \\
\hline HS48 & 22727,28 & $\mathrm{a}$ & 11440,03 & $\mathrm{~b}$ & 15403,71 & $\mathrm{a}$ & 6195,32 & $\mathrm{a}$ \\
\hline L55 & 11776,50 & $\mathrm{c}$ & 1904,26 & $\mathrm{e}$ & 8691,28 & $\mathrm{~b}$ & 2523,44 & $\mathrm{~b}$ \\
\hline HS56 & 18830,30 & $\mathrm{a}$ & 8387,21 & $\mathrm{c}$ & 12801,73 & $\mathrm{a}$ & 4539,07 & $\mathrm{a}$ \\
\hline HS57 & 17137,04 & $\mathrm{~b}$ & 8020,48 & $\mathrm{c}$ & 12371,50 & $\mathrm{a}$ & 5320,32 & $\mathrm{a}$ \\
\hline HS58 & 17885,22 & $\mathrm{a}$ & 6582,87 & $\mathrm{~d}$ & 12351,05 & $\mathrm{a}$ & 5960,94 & $\mathrm{a}$ \\
\hline L66 & 7729,52 & $\mathrm{c}$ & 0,00 & $\mathrm{f}$ & 5258,55 & $\mathrm{c}$ & 0,00 & $\mathrm{~b}$ \\
\hline HS67 & 17444,71 & $\mathrm{~b}$ & 6557,97 & $\mathrm{~d}$ & 9778,42 & $\mathrm{a}$ & 3070,32 & b \\
\hline HS68 & 18509,26 & $\mathrm{a}$ & 8557,55 & $\mathrm{c}$ & 11202,09 & $\mathrm{a}$ & 2695,32 & $\mathrm{~b}$ \\
\hline L77 & 8320,32 & $\mathrm{c}$ & 0,00 & $\mathrm{f}$ & 7536,55 & $\mathrm{~b}$ & 2343,76 & $\mathrm{~b}$ \\
\hline HS78 & 16208,33 & $\mathrm{~b}$ & 8156,15 & $\mathrm{c}$ & 11440,19 & $\mathrm{a}$ & 4601,57 & $\mathrm{a}$ \\
\hline L88 & 10565,22 & $\mathrm{c}$ & 3109,89 & $\mathrm{e}$ & 7635,84 & $\mathrm{~b}$ & 1734,38 & $\mathrm{~b}$ \\
\hline
\end{tabular}

Médias seguidas pela mesma letra na coluna pertencem ao mesmo agrupamento, pelo teste de Scott-Knott, a 5\% de probabilidade. 
Tabela 3 - Valores médios da altura de plantas, porcentagem de massa, cor dos grãos, diâmetro e comprimento das espigas, considerando a média das características nos dois experimentos. UFLA, Lavras, MG, 2007.

\begin{tabular}{|c|c|c|c|c|c|c|}
\hline Genótipos & ALT (m) & MASSA (\%) & COR (escala) & DIAM $(\mathrm{cm})$ & COMP & $(\mathrm{cm})$ \\
\hline L11 & 1,89 a & $37,50 \mathrm{~b}$ & $2 b$ & $3,52 \mathrm{c}$ & 14,55 & $\mathrm{~b}$ \\
\hline HS12 & 2,09 a & $41,13 \mathrm{~b}$ & $2 \mathrm{~b}$ & 4,55 a & 16,98 & $\mathrm{a}$ \\
\hline HS13 & 2,16 a & 49,74 a & $4 \mathrm{a}$ & $4,25 \mathrm{~b}$ & 16,83 & $\mathrm{a}$ \\
\hline HS14 & 2,06 a & 34,82 b & $2 \mathrm{~b}$ & $4,29 \mathrm{~b}$ & 18,33 & $\mathrm{a}$ \\
\hline HS15 & $2,04 \mathrm{a}$ & 46,09 a & $3 \mathrm{a}$ & $4,40 \quad b$ & 17,67 & $\mathrm{a}$ \\
\hline HS16 & 2,11 a & $36,71 \quad b$ & $2 \mathrm{~b}$ & $4,17 \quad b$ & 16,18 & $\mathrm{~b}$ \\
\hline HS17 & 2,07 a & $44,02 \mathrm{a}$ & $3 \mathrm{~b}$ & $4,01 \quad b$ & 17,00 & $\mathrm{a}$ \\
\hline HS18 & 1,98 a & 47,17 a & $3 \mathrm{a}$ & $4,41 \quad b$ & 15,92 & $\mathrm{~b}$ \\
\hline L22 & $1,24 \mathrm{c}$ & $11,75 \mathrm{~d}$ & $2 \mathrm{~b}$ & $3,82 \mathrm{c}$ & 8,51 & e \\
\hline HS23 & $1,90 \mathrm{a}$ & $34,00 \mathrm{~b}$ & $2 \mathrm{~b}$ & 4,71 a & 14,86 & $\mathrm{~b}$ \\
\hline HS24 & $1,92 \mathrm{a}$ & $37,88 \mathrm{~b}$ & $2 \mathrm{~b}$ & 4,59 a & 16,52 & $\mathrm{a}$ \\
\hline HS25 & 1,86 a & 49,91 a & $3 \mathrm{~b}$ & 4,77 a & 17,27 & $\mathrm{a}$ \\
\hline HS26 & 2,04 a & 46,27 a & $3 a$ & $4,39 \quad b$ & 15,98 & $\mathrm{~b}$ \\
\hline HS27 & 2,05 a & 51,07 a & $2 \mathrm{~b}$ & 4,70 a & 17,45 & $\mathrm{a}$ \\
\hline HS28 & 2,17 a & $39,34 \mathrm{~b}$ & $3 \mathrm{a}$ & $4,33 \mathrm{~b}$ & 15,91 & $\mathrm{~b}$ \\
\hline L33 & $1,48 \quad b$ & $35,54 \mathrm{~b}$ & $2 \mathrm{~b}$ & $3,66 \mathrm{c}$ & 16,00 & $\mathrm{~b}$ \\
\hline HS34 & $2,00 \quad \mathrm{a}$ & 47,75 a & $3 \mathrm{a}$ & $4,25 \mathrm{~b}$ & 17,44 & $\mathrm{a}$ \\
\hline HS35 & $2,00 \mathrm{a}$ & $36,02 \mathrm{~b}$ & $4 \mathrm{a}$ & $4,08 \mathrm{~b}$ & 16,07 & $\mathrm{~b}$ \\
\hline HS36 & 2,02 a & $37,37 \mathrm{~b}$ & $3 \mathrm{a}$ & $4,26 \mathrm{~b}$ & 15,23 & $\mathrm{~b}$ \\
\hline HS37 & $1,93 \mathrm{a}$ & 46,22 a & $4 \mathrm{a}$ & $4,15 \mathrm{~b}$ & 16,78 & $\mathrm{a}$ \\
\hline HS38 & $1,67 \mathrm{~b}$ & $38,71 \mathrm{~b}$ & $1 \mathrm{~b}$ & $3,56 \mathrm{c}$ & 11,55 & d \\
\hline L44 & $1,28 \mathrm{c}$ & $14,92 \mathrm{~d}$ & $2 \mathrm{~b}$ & $3,66 \mathrm{c}$ & 10,62 & d \\
\hline HS45 & $1,95 \mathrm{a}$ & 47,26 a & $3 \mathrm{a}$ & $4,22 \mathrm{~b}$ & 18,91 & $\mathrm{a}$ \\
\hline HS46 & $1,86 \mathrm{a}$ & 54,90 a & $3 \mathrm{~b}$ & $4,54 \mathrm{a}$ & 16,63 & $\mathrm{a}$ \\
\hline HS47 & $1,83 \mathrm{a}$ & 49,71 a & $2 \mathrm{~b}$ & $4,23 \mathrm{~b}$ & 17,15 & $\mathrm{a}$ \\
\hline HS48 & 2,14 a & 53,23 a & $2 \mathrm{~b}$ & 4,57 a & 17,58 & $\mathrm{a}$ \\
\hline L55 & $1,55 \mathrm{~b}$ & $31,42 \mathrm{~b}$ & $4 \mathrm{a}$ & $3,67 \mathrm{c}$ & 15,23 & $\mathrm{~b}$ \\
\hline HS56 & $1,85 \mathrm{a}$ & 52,27 a & $4 \mathrm{a}$ & $4,35 \mathrm{~b}$ & 16,78 & $\mathrm{a}$ \\
\hline HS57 & $1,86 \mathrm{a}$ & 43,36 a & $3 \mathrm{a}$ & $4,11 \mathrm{~b}$ & 17,17 & $\mathrm{a}$ \\
\hline HS58 & $2,05 \mathrm{a}$ & 49,91 a & $4 \mathrm{a}$ & $4,15 \mathrm{~b}$ & 16,37 & $\mathrm{~b}$ \\
\hline L66 & $1,24 \mathrm{c}$ & $14,38 \mathrm{~d}$ & $2 \mathrm{~b}$ & $3,50 \mathrm{c}$ & 10,91 & d \\
\hline HS67 & 1,72 a & $28,68 \mathrm{c}$ & $2 \mathrm{~b}$ & $4,19 \mathrm{~b}$ & 16,25 & $\mathrm{~b}$ \\
\hline HS68 & $1,97 \mathrm{a}$ & $45,97 \mathrm{a}$ & $3 \mathrm{~b}$ & $4,31 \mathrm{~b}$ & 15,89 & $\mathrm{~b}$ \\
\hline L77 & $1,17 \mathrm{c}$ & $20,00 \mathrm{~d}$ & $2 \mathrm{~b}$ & $3,56 \mathrm{c}$ & 12,91 & $\mathrm{c}$ \\
\hline HS78 & $1,91 \mathrm{a}$ & $42,06 \mathrm{~b}$ & $2 \mathrm{~b}$ & $4,08 \quad b$ & 16,92 & $\mathrm{a}$ \\
\hline L88 & $1,46 \mathrm{~b}$ & $34,79 \mathrm{~b}$ & $1 \mathrm{~b}$ & $3,69 \mathrm{c}$ & 14,29 & $\mathrm{~b}$ \\
\hline Média & 1,85 & 39,77 & 2,47 & 4,16 & 15,74 & \\
\hline
\end{tabular}

Médias seguidas pela mesma letra na coluna pertencem ao mesmo agrupamento, pelo teste de Scott-Knott, a 5\% de probabilidade. 
A herdabilidade variou de $97 \%$ (PEC) a $73 \%$ (COR) em Lavras e 88,99\% (COMP) a 58,80\% (DIAM) em Ijaci (Tabela 4). Em milho, nas condições brasileiras, diversos autores consideram valores da $C V_{g}$ acima de $7 \%$, como um bom indicador do potencial genético de germoplasma para o melhoramento. Exceto para o diâmetro de espigas em Ijaci, o valor do $C V_{g}$ foi superior a esse valor para todas as características avaliadas.

Nota-se que, em Lavras, a menor estimativa de $h^{2}$ foi obtida na característica COR $(73,06 \%)$ e em Ijaci pela característica DIAM $(58,80 \%)$ (Tabela 4). Porém, os valores obtidos para as demais características, nos dois locais, foram de alta magnitude. Assim, a seleção poderá ser feita com grande possibilidade de sucesso, para as diferentes características de milho-verde, mesmo para características quantitativas, que normalmente apresentam valores mais baixos para $h^{2}$.

Variação semelhante foi observada na razão entre o coeficiente de variação genética e ambiental $\left(C V_{g} / C V_{e}\right)$, os quais retratam uma situação mais favorável para seleção nas características PEE, PEC e COMP, em Lavras, e ALT, PEE e COMP, em Ijaci (Tabela 4).

É possível antever a possibilidade de sucesso na seleção em programas de melhoramento, visando à produção de milho verde em função das altas magnitudes das estimativas da herdabilidade e da variância genética obtidas para a maioria das características avaliadas.

A obtenção da correlação entre características de interesse de milho-verde, pode facilitar a seleção e diminuir o trabalho do melhorista na seleção de híbridos promissores que possam atender às exigências do consumidor.

As estimativas do coeficiente de correlação genética entre as sete características avaliadas estão apresentadas na Tabela 5. Nota-se que houve correlação positiva e significativa para a maioria das características. Porém, a característica COR apresentou valores baixos e não significativos para todos os pares de características em queessa característica estava incluída. Vale ressaltar

Tabela 4 - Estimativas das variâncias fenotípicas $\left(\sigma_{F}^{2}\right)$, variâncias ambientais $\left(\sigma_{E}^{2}\right)$, variâncias genéticas $\left(\sigma_{G}^{2}\right)$, herdabilidades $\left(\mathrm{h}^{2}\right)$, coeficientes de variação genética $\left(\mathrm{CV}_{\mathrm{g}}\right)$, razão entre coeficientes de variação genética e ambiental $\left(\mathrm{CV}_{\mathrm{g}} / \mathrm{CV}_{\mathrm{e}}\right)$ e o erro padrão da média das sete características avaliadas em Lavras, MG e Ijaci, MG. UFLA, Lavras, MG, 2007 .

\begin{tabular}{llllllll}
\hline \multicolumn{7}{c}{ LAVRAS } \\
\hline & ALT & PEE & PEC & MASSA & COR & DIAM & COMP \\
\hline$\sigma_{F}^{2}$ & 0,113 & 17291436,54 & 12650103,51 & 147,23 & 0,762 & 0,199 & 5,23 \\
$\sigma_{E}^{2}$ & 0,014 & 1460601,05 & 358556,18 & 19,87 & 0,205 & 0,023 & 0,521 \\
$\sigma_{G}^{2}$ & 0,098 & 15830835,50 & 12291547,33 & 127,36 & 0,557 & 0,176 & 4,71 \\
$\mathrm{~h}^{2}$ & 86,94 & 91,55 & 97,16 & 86,50 & 73,07 & 88,41 & 90,04 \\
$\mathrm{CV}_{\mathrm{g}}$ & 14,44 & 24,79 & 49,30 & 26,67 & 33,80 & 9,68 & 13,00 \\
$\mathrm{CV}_{\mathrm{g}} / \mathrm{CV} \mathrm{e}$ & 1,82 & 2,33 & 4,14 & 1,79 & 1,16 & 1,95 & 2,12 \\
$\mathrm{Erro}^{2}$ & 0,041 & 508,12 & 422,03 & 1,51 & 0,115 & 0,055 & 0,281 \\
\hline & & & & $\mathrm{IJACI}$ & & & \\
\hline$\sigma_{F}^{2}$ & $\mathrm{ALT}$ & $\mathrm{PEE}$ & $\mathrm{PEC}$ & $\mathrm{MASSA}$ & $\mathrm{COR}$ & $\mathrm{DIAM}$ & $\mathrm{COMP}$ \\
$\sigma_{E}^{2}$ & 0,069 & 7183688,97 & 3440290,13 & 141,74 & 0,691 & 0,124 & 6,05 \\
$\sigma_{G}^{2}$ & 0,009 & 799279,95 & 810644,68 & 21,47 & 0,173 & 0,051 & 0,667 \\
$\mathrm{~h}^{2}$ & 0,061 & 6384409,02 & 2629645,44 & 120,26 & 0,517 & 0,073 & 5,39 \\
$\mathrm{CV}$ & 87,92 & 88,87 & 76,43 & 84,84 & 74,87 & 58,80 & 88,99 \\
$\mathrm{CV}$ & 16,18 & 23,15 & 41,84 & 29,45 & 30,28 & 6,78 & 15,71 \\
$\mathrm{Erro}_{\mathrm{g}}$ & 1,91 & 1,99 & 1,27 & 1,67 & 1,22 & 0,84 & 2,01 \\
\hline & 0,033 & 334,38 & 248,99 & 1,49 & 0,11 & 0,49 & 0,307 \\
\hline
\end{tabular}


que a cor é uma característica de fácil avaliação e sua determinação não interferirá na agilidade do processo de seleção de híbridos promissores.

Observou-se correlação alta e significativa entre a característica PEE com PEC, DIAM e COMP. Assim, pode-se afirmar que genótipos com maior produção de espigas empalhadas, apresentam maior produção de espigas comerciais, assim como maior diâmetro e comprimento de espigas (Tabela 5). A alta estimativa da correlação facilita o processo de seleção de híbridos visando a atender as exigências do mercado, ou seja, permite ao melhorista selecionar ou reduzir, o número de características a serem avaliadas, sem a necessidade de medições adicionais. Resultados semelhantes foram obtidos por Oliveira et al. (1987) e Pinho et al. (2001).

Genótipos com espigas de maior diâmetro não indicam necessariamente genótipos que produzem espigas com maior porcentagem de massa (MASSA), o que pode ser verificada pela baixa estimativa obtida entre essas características (Tabela 5). Dessa forma, em experimentos futuros com milho-verde deve-se avaliar a porcentagem de massa de cada genótipo, independentemente da avaliação do diâmetro das espigas.

O método do índice de seleção baseado na soma de postos facilita a decisão do melhorista sobre quais genótipos devem ser selecionados e quais devem ser descartados. O método é de fácil obtenção e os pesos podem ser atribuídos de acordo com o que o melhorista deseja nos genótipos, a fim de prosseguir com o programa.

Pelos resultados apresentados na Tabela 6 , é possível inferir quais híbridos apresentaram o melhor desempenho, simultaneamente, para todas as características avaliadas. Os híbridos HS48, HS27, HS24, HS25, HS12 e HS45 acumularam um maior número de características desejáveis, em um único genótipo.

Os híbridos HS35, HS36 e HS38 apresentaram desempenho inferior em relação aos demais híbridos avaliados no experimento, considerando simultaneamente as características (Tabela 6). Vale ressaltar que os pesos econômicos utilizados foram capazes de distinguir os híbridos mais promissores e podem ser utilizados como critério de seleção em programas de melhoramento de milho-verde.

Tabela 5 - Estimativas do coeficiente de correlação genética $\left(\mathrm{r}_{\mathrm{G}}\right)$ entre altura de plantas (ALT), produtividade de espigas empalhadas (PEE), produtividade de espigas comerciais (PEC), porcentagem de massa (MASSA), cor dos grãos (COR), diâmetro (DIAM) e comprimento das espigas (COMP). UFLA, Lavras, MG, 2007.

\begin{tabular}{ccccccc}
\hline CARACTERÍSTICAS & PEE & PEC & MASSA & COR & DIAM & COMP \\
\hline ALT & $0,58^{*}$ & $0,43^{*}$ & $0,25^{\text {n.s }}$ & $0,29^{\text {n.s }}$ & $0,34^{\text {n.s }}$ & $0,44^{\text {n.s }}$ \\
PEE & & $0,88^{* *}$ & $0,30^{* *}$ & $0,05^{\text {n.s }}$ & $0,81^{* *}$ & $0,77^{* *}$ \\
PEC & & & $0,45^{\text {n.s }}$ & $-0,21^{\text {n.s }}$ & $0,70^{* *}$ & $0,80^{* *}$ \\
MASSA & & & & $0,38^{\text {n.s }}$ & $0,22^{\text {n.s }}$ & $0,43^{\text {n.s }}$ \\
COR & & & & & $-0,11^{\text {n.s }}$ & $0,18^{\text {n.s }}$ \\
DIAM & & & & & & $0,38^{\text {n.s }}$ \\
\hline
\end{tabular}

* e ${ }^{* *}$ - significativo e altamente significativo ao teste $\mathrm{t}$; ${ }^{\text {n.s }}$ - não significativo. 
Tabela 6 - Classificação de híbridos experimentais, com base na soma de postos, envolvendo sete características de interesse agronômico e diferentes pesos econômicos. UFLA, Lavras, MG, 2007.

\begin{tabular}{ccccccccc}
\hline HÍBRIDOS & ALT & PEE & PEC & MASSA & COR & DIAM & COMP & ÍNDICE \\
\hline HS48 & 0,6 & 28 & 28 & 8,1 & 10 & 12 & 12,5 & 86,7 \\
HS27 & 1,8 & 27 & 27 & 7,5 & 10 & 13 & 12 & 86,3 \\
HS24 & 3,8 & 25 & 26 & 2,1 & 12 & 12,5 & 6 & 81,4 \\
HS25 & 4,6 & 23 & 22 & 6,9 & 8 & 14 & 11 & 78,5 \\
HS12 & 1 & 24 & 24 & 3 & 10 & 11,5 & 9 & 73,5 \\
HS45 & 3,4 & 26 & 25 & 5,7 & 6 & 5 & 14 & 71,1 \\
HS15 & 2 & 22 & 23 & 4,5 & 6 & 10 & 13 & 67,5 \\
HS23 & 4,2 & 18 & 19 & 0,6 & 10 & 13,5 & 1 & 65,3 \\
HS14 & 1,4 & 21 & 21 & 0,9 & 12 & 7,5 & 13,5 & 63,8 \\
HS46 & 4,4 & 11 & 16 & 8,4 & 8 & 11 & 6,5 & 58,8 \\
HS56 & 5 & 19 & 13 & 7,8 & 2 & 9 & 7 & 55,8 \\
HS47 & 5,2 & 6 & 18 & 6,3 & 10 & 5,5 & 10 & 51,0 \\
HS26 & 2,2 & 16 & 9 & 5,1 & 6 & 9,5 & 3,5 & 47,8 \\
HS18 & 3 & 17 & 5 & 5,4 & 6 & 10,5 & 3 & 46,9 \\
HS57 & 4,8 & 12 & 17 & 3,6 & 6 & 2,5 & 10,5 & 45,9 \\
HS34 & 2,8 & 13 & 12 & 6 & 6 & 6 & 11,5 & 45,8 \\
HS28 & 0,2 & 20 & 8 & 2,7 & 6 & 8,5 & 2,5 & 45,4 \\
HS17 & 1,2 & 10 & 20 & 3,9 & 8 & 1 & 9,5 & 44,1 \\
HS68 & 3,2 & 14 & 6 & 4,2 & 8 & 8 & 2 & 43,4 \\
HS78 & 4 & 8 & 11 & 3,3 & 12 & 1,5 & 8,5 & 39,8 \\
HS58 & 1,6 & 15 & 10 & 7,2 & 2 & 3 & 5,5 & 38,8 \\
HS13 & 0,4 & 5 & 14 & 6,6 & 4 & 6,5 & 8 & 36,5 \\
HS37 & 3,6 & 3 & 15 & 4,8 & 4 & 3,5 & 7,5 & 33,9 \\
HS16 & 0,8 & 9 & 7 & 1,5 & 10 & 4 & 4,5 & 32,3 \\
HS67 & 5,4 & 7 & 3 & 0,3 & 10 & 4,5 & 5 & 30,2 \\
HS38 & 5,6 & 1 & 1 & 2,4 & 14 & 0,5 & 0,5 & 24,5 \\
HS36 & 2,4 & 2 & 2 & 1,8 & 6 & 7 & 1,5 & 21,2 \\
HS35 & 2,6 & 4 & 4 & 1,2 & 2 & 2 & 4 & 15,8 \\
\hline
\end{tabular}

\section{CONCLUSÕES}

A avaliação da produtividade de espigas empalhadas (PEE) é suficiente para definir quais híbridos apresentam o melhor desempenho para a produtividade de espigas comerciais (PEC), diâmetro (DIAM) e comprimento de espigas (COMP). Isso indica que as características PEC, DIAM e COMP podem ser descartadas da avaliação em programas de melhoramento de milho- verde.

Os híbridos HS48, HS27, HS24, HS25, HS12 e HS45 acumularam um maior número de características desejadas em um único genótipo, com base no índice de seleção baseado na soma de postos, sendo os mais promissores para a produção de milho-verde.

Os pesos econômicos utilizados foram capazes de distinguir e identificar os híbridos mais promissores para a produção de milho-verde e podem ser utilizados como critério de seleção em programas de melhoramento.

\section{AGRADECIMENTOS}

Os autores agradecem a FAPEMIG (Fundação de Apoio a Pesquisa do Estado de Minas Gerais pelo apoio financeiro para a condução desta pesquisa. 


\section{REFERÊNCIAS BIBLIOGRÁFICAS}

ALBUQUERQUE, C.J.B.; PINHO, R.G. von; BORGES, I.D.; SOUZA FILHO, A.X.; FIORINI, I.V. Desempenho de híbridos experimentais e comerciais de milho para produção de milho verde. Ciência e Agrotecnologia, Lavras, v.32, n.3, p.768-775, 2008.

BORDALLO, P.N.; PEREIRA, M.G.; AMARAL JÚNIOR, A.T.; GABRIEL, A.P.C. Análise dialélica de genótipos de milho doce e comum para caracteres agronômicos e proteína total. Horticultura Brasileira, Brasília, v.23, n.1, p.123-127, jan./mar. 2005.

CRUZ, C.D.; REGAZZI, A.J. Modelos biométricos aplicados ao melhoramento genético. 2.ed. Viçosa, MG: UFV, 2001. 390p.

MATOS, M.J.L.F.; TAVARES, S.A.; SANTOS, F.F. dos; MELO, M.F. de; LANA, M.M. Milho verde. Brasília: Embrapa, 2006. Disponível em: <hțtp:///www.cnphi. embrapa.br/paginas/dicas_ao_consumidor/ milho_verde.htm>. Acesso em: 16 jun. 2006.

MULAMBA, N.N.; MOCK, J.J. Improvement of yield potential of the Eto Blanco maize (Zea mays L.) population by breeding for plant traits. Egyptian Journal of Genetics and Cytology, Alexandria, v.7, n.1, p.40-51, 1978.

OLIVEIRA, L.A.A. de; GROSZMAN, A.; COSTA, R.A. da. Caracteres da espiga de cultivares de milho no estádio verde. Pesquisa Agropecuária Brasileira, Brasília, v.22, n.6, p.587-592, jun. 1987.

PEREIRA FILHO, I.A.; CRUZ, J.C.; GAMA, E.E.G. Cultivares para o consumo verde. In: PEREIRA FILHO, I.A. (Ed.). O cultivo do milho verde. Brasília: Embrapa Informação Tecnológica, 2003. 204p.

PINHO, R.G. von; PAIVA JUNIOR, M.C.; PINHO, É.V.R. von; RESENDE, S.G. Desempenho de cultivares para a produção de milho verde em diferentes épocas e densidades de semeadura. Ciência e Agrotecnologia, Lavras, v.25, n.5, p.2351247, 2001.

VENKOVSKY, R.; BARRIGA, P. Genética biométrica no fitomelhoramento. Ribeirão Preto: Sociedade Brasileira de Genética, 1992. 486p. 